\title{
Novel heterozygous MCCC1 mutations identified in a patient with 3-methylcrotonyl-coenzyme A carboxylase deficiency
}

\author{
Yoon-Myung Kim¹, Go Hun Seo, Gu-Hwan Kim², Han-Wook Yoo, and Beom Hee Lee ${ }^{1, *}$ \\ ${ }^{1}$ Department of Pediatrics, Asan Medical Center Children's Hospital, University of Ulsan College of Medicine, Seoul, Korea \\ ${ }^{2}$ Medical Genetics Center, Asan Medical Center Children's Hospital, University of Ulsan College of Medicine, Seoul, Korea
}

\begin{abstract}
Isolated 3-methylcrotonyl-CoA carboxylase deficiency is an autosomal recessive disorder affecting leucine metabolism; it is one of the most common inborn metabolic diseases detected in newborn screening. Mutations in the genes MCCC1 or MCCC2 cause a defect in the enzyme 3-methylcrotonyl-CoA carboxylase, with MCCC2 mutations being the form predominantly reported in Korea. The majority of infants identified by neonatal screening usually appear to be asymptomatic and remain healthy; however, some patients have been reported to exhibit mild to severe metabolic decompensation and neurologic manifestations. Here we report the clinical features of a patient with asymptomatic 3-methylcrotonyl-CoA carboxylase deficiency and novel heterozygous MCCC1 mutations.
\end{abstract}

Key words: 3-Methylcrotonyl-CoA carboxylase deficiency, 3-Methylcrotonylglycinuria, MCCC1.

\section{Introduction}

Isolated 3-methylcrotonyl-CoA carboxylase (3-MCC) deficiency (OMIM\# 210200 and 210210) is an autosomal recessive disorder impacting leucine metabolism. It is one of the most common inborn metabolic errors and is detected via newborn screening with a frequency of approximately 1 in 36,000 births [1].

3-MCC is a biotin-dependent carboxylase consisting of $\alpha$ and $\beta$ subunits encoded by $M C C C 1$ and $M C C C 2$, respectively [2]. MCCC 1 has 19 exons and is located in the chromosomal region 3q25-q27, whereas MCCC2 consists of 17 exons and is found in the region $5 q 12-q 13$ [2]. Mutations in one of these two genes cause defects in 3-MCC and thus inhibit the conversion of 3-methylcrotonyl-CoA to 3-methylglutaconyl-CoA. Instead,
3-methylcrotonyl-CoA accumulates and is eventually converted into 3-hydroxyisovaleric acid and 3-methylcrotonylglycine, which are typically elevated in this disease. MCCC2 mutations were reported to be 1.7 times more common than MCCC1 mutations, as found in a cohort study of 53 newborns [3]. Of the genetic mutations reported in Korean patients, 75\% have been found within MCCC2 $[4,5]$. Infants carrying a mutation identified by neonatal screening usually appear to be asymptomatic and remain healthy. However, some patients are reported to present with hypotonia, encephalopathy, seizure, failure to thrive, cardiomyopathy, and late onset severe metabolic decompensation with metabolic acidosis and hypoglycemia [1,6-9].

In this report, we present the clinical characteristics, laboratory findings and molecular analysis of a patient possessing novel heterozygous mutations of $M C C C 1$.

\footnotetext{
Received: 23 April 2017, Revised: 19 May 2017, Accepted: 21 May 2017, Published: 30 June 2017

*Corresponding author: Beom Hee Lee, M.D., Ph.D.

Department of Pediatrics, Asan Medical Center Children's Hospital, University of Ulsan College of Medicine, 88 Olympic-ro 43-gil, Songpa-gu, Seoul 05505, Korea.

Tel: +82-2-3010-5950, Fax: +82-2-473-3725, E-mail: bhlee@amc.seoul.kr

Conflict of interest: The authors declare that they do not have any conflicts of interest.

(c) This is an open-access article distributed under the terms of the Creative Commons Attribution Non-Commercial License (http://creativecommons.org/licenses/by-nc/4.0/) which permits unrestricted non-commercial use, distribution, and reproduction in any medium, provided the original work is properly cited.

(c) Copyright 2017 by the Korean Society of Medical Genetics and Genomics

www.e-kjgm.org
} 


\section{Case}

A 26 day-old baby was referred to Asan Medical Center with abnormalities found through tandem mass spectrometry analysis of blood samples. He was born in the 39th week of gestation with a weight of $3.45 \mathrm{~kg}$ to non-consanguineous parents; he was their first child and there was no specific family history of disease. There were no perinatal problems and the child appeared healthy. He was fed with both breast milk and artificial formula. On the third day of life, tandem mass spectrometry was performed, revealing that the level of 3-hydroxyisovalerylcarnitine $(\mathrm{C} 5-\mathrm{OH})$ was $3.84 \mu \mathrm{mol} / \mathrm{L}-$ the reference range is $<0.7$ $\mu \mathrm{mol} / \mathrm{L}$. The analysis was repeated twice: the subsequent $\mathrm{C} 5-\mathrm{OH}$ levels were $6.34 \mu \mathrm{mol} / \mathrm{L}$ and $5.38 \mu \mathrm{mol} / \mathrm{L}$, respectively. The leucine concentration was within the normal range and the level of free carnitine was $5.21 \mu \mathrm{mol} / \mathrm{L}$, far below the reference range of 26-76 $\mu \mathrm{mol} / \mathrm{L}$.

Due to his persistently elevated level of $\mathrm{C} 5-\mathrm{OH}$, the patient was referred to our clinic for further metabolic workup. He was not dysmorphic, appeared healthy, and was physically active. He had no history of poor oral intake, vomiting, lethargy, or seizure. Height, weight, and head circumference was $53.7 \mathrm{~cm}, 3.9 \mathrm{~kg}$, and $37.5 \mathrm{~cm}$, respectively; each value was within the 75th-90th percentile of its corresponding field. Tandem mass spectrometry, urine organic acid, serum organic acid, serum amino acid and acylcarnitine analyses were performed, while the boy was fed with leucine-restricted formula until the test results were ready. Blood chemistry levels-including ammonia and lactate content-were within normal limits. Brain sonography appeared to be normal. $\mathrm{C} 5-\mathrm{OH}$ was measured as $7.06 \mu \mathrm{mol} / \mathrm{L}$ by tandem mass spectrometry and $8.617 \mu \mathrm{mol} / \mathrm{L}$ by acylcarnitine profiling.
The level of free carnitine was determined to be $12.271 \mu \mathrm{mol} /$ L. Serum amino acid analysis yielded non-specific findings, with valine, leucine, and isoleucine levels all within their normal ranges. However, the concentration of $\mathrm{C} 5-\mathrm{OH}$ was $30.8 \mu \mathrm{mol} / \mathrm{L}$ (reference range, 1.3-7.0 $\mu \mathrm{mol} / \mathrm{L}$ ) in serum and $996.9 \mathrm{mmol} / \mathrm{mol}$ creatinine $(\mathrm{Cr}$; reference range, $1.0-20.0 \mathrm{mmol} / \mathrm{mol} \mathrm{Cr})$ in urine, while that of 3-methylcrotonylglycine was $2108.2 \mathrm{mmol} / \mathrm{mol} \mathrm{Cr}$ (reference range, $0 \mathrm{mmol} / \mathrm{mol} \mathrm{Cr}$ ) in urine.

The cause of these abnormal levels was suspected to be 3-MCCdeficiency; as such, the MCCC2 gene was sequenced, using genomic DNA from peripheral blood leukocytes drawn from the patient. All coding exons and exon-intron boundaries of the gene were individually amplified through polymerase chain reaction (PCR) by using primers complimentary to the flanking regions of the sequences of interest; the amplified PCR products were then sequenced. Analysis of MCCC2 identified no mutations, so we then sequenced MCCC1. Two heterozygous MCCC1 mutations were identified: c. $1 \mathrm{~A}>\mathrm{G}(\mathrm{p} .0)$ in exon 1 and c.581_598delinsTTA (p.His194Leufs*2) in exon 6 (Fig. 1), neither of which has been previously reported [10-13]. We finally diagnosed this patient as having 3-MCC deficiency stemming from these two heterozygous $\mathrm{MCCC} 1$ mutations. Gene sequencing of the parents was not performed. The patient is now 8 months old and is being fed with a mixture of leucine-free and general formula (74 mg of leucine/ $\mathrm{kg} /$ day). He is still asymptomatic and in good health: he exhibits normal growth and development and is able to sit alone and stand with support.

\section{Discussion}

We identified a patient with novel heterozygous mutations-

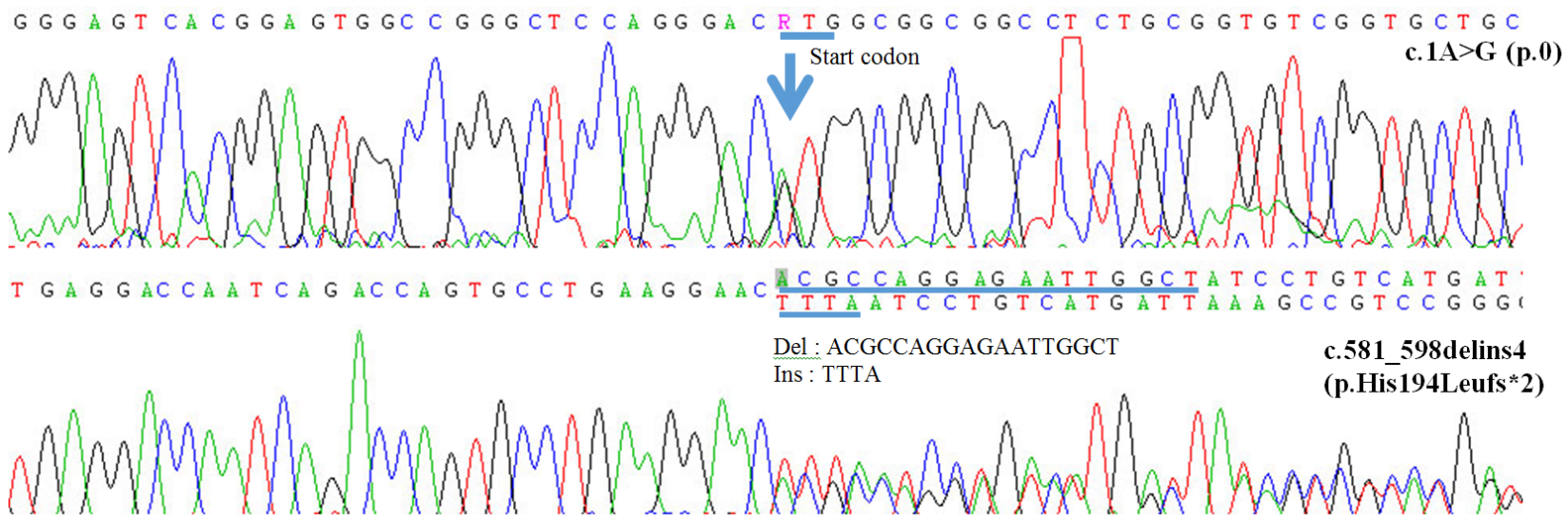

c. [1 A>G];[581_598delinsTTTA] (p.[0]; [His194Leufs*2]), novel

$\mathrm{R}=\mathrm{A}$ and $\mathrm{G}$

Fig. 1. Partial MCCC1 gene sequence showing novel heterozygote mutations including c.1A>G (p.0) and c.581_598delinsTTTA (p.His194Leuf^²). 
c. 1 A >G (p.0) and c.581_598delinsTTA (p.His194Leufs*2)of $\mathrm{MCCC} 1$ who is currently 8 months old and asymptomatic. Neither mutation is found in the general population; both are expected to be deleterious because c. $1 \mathrm{~A}>\mathrm{G}$ is located in the start codon and p.His194Leufs 2 is a frameshift mutation.

The genotype-phenotype correlation of $\mathrm{MCCC} 1$ and $\mathrm{MCCC} 2$ is not clearly understood $[3,14]$. No reports have revealed any difference in phenotype between $M C C C 1$ and $M C C C 2$ genotypes. Mutations have been reported in both symptomatic and asymptomatic patients, and the degree of phenotypic severity is known to vary among symptomatic individuals [14]. Previous reports suggested that elements other than genotype, such as environmental factors, may have a major influence on the phenotype of isolated 3-MCC deficiency [14]. The clinical phenotype of the disease is heterogeneous and ranges from asymptomatic to lethal [3]. Severe phenotypes include late onset metabolic decompensation, severe hypoglycemia, early onset necrotizing encephalopathy and recurrent status epilepticus [7-9]. Further metabolic profiling-including urine organic acid, plasma acylcarnitine and biotinidase analyses-is recommended for all infants who test positive for the deficiency [1]. Although the results may be within normal ranges, standard illnesses can trigger metabolic stress and thus induce a late onset symptomatic phenotype.

In general, most isolated 3-MCC deficiency-positive newborns subjected to screening appear clinically normal and healthy. Symptoms such as vomiting, ketosis, poor oral intake, irritability, lethargy and hypotonia were reported in up to 15\% of patients, but the majority (92.5\%) of subjects showed completely appropriate age-matched development [15]. In some cases, healthy, affected mothers have been identified through their children's newborn screenings; and $\mathrm{C} 5-\mathrm{OH}$ is known to be elevated in an affected mother's breast milk [16]. An Israeli study described a group of 36 individuals initially identified by a newborn screening program as possessing isolated 3-MCC deficiency; subsequent testing revealed that 20 of those cases were maternal-the infants lacked the disease but had metabolic profiles reflecting those of their affected mothers. The mean concentration of free carnitine (CO) in unaffected newborns from affected mothers was significantly lower compared with that of affected newborns (7.27 $\mu \mathrm{M}$ vs. $18.97 \mu \mathrm{M}, P=0.0009)$. C5- 0 H starts to accumulate only after birth in patients with the disease, causing only a modest decrease in initial C0 levels [17]; thus, the measure of perinatal $\mathrm{CO}$ levels may be useful in distinguishing maternally influenced elevation of $\mathrm{C} 5-\mathrm{OH}$ [17]. Of the 20 mothers diagnosed with maternal 3-MCC deficiency in the previous report,
19 were reported to be completely asymptomatic except for one who had been diagnosed with childhood hypotonia, demonstrating the low proportion of symptomatic patients [17]. Considering the low frequency of symptomatic patients and the lack of reliable parameters for predicting the course of the disease, some researchers have suggested excluding 3-MCC deficiency from the newborn screening program [17].

In the concern of susceptibility to metabolic stress during illness, it is recommended that patients with isolated 3-MCC deficiency avoid long-term fasting and obtain an adequate calorie intake [1]. A leucine-restricted diet in early infancy may reduce the burden of metabolic disturbances, but persistent dietary restrictions are not necessary for asymptomatic patients. The efficacy of leucine-restricted diets is unknown $[1,18]$ and further evaluation is needed to clarify whether regular protein diets increase metabolic risk. Supplementation of carnitine may be beneficial in increasing serum carnitine level, but the clinical efficacy of this has not been demonstrated $[1,19]$.

We report an asymptomatic 3-MCC deficiency patient with novel heterozygous MCCC1 mutations. Further studies are needed to clarify the necessity of screening and management, as well as the true nature of the genotype-phenotype relationship and long-term outcomes.

\section{References}

1. Arnold GL, Koeberl DD, Matern D, Barshop B, Braverman N, Burton $B$, et al. A Delphi-based consensus clinical practice protocol for the diagnosis and management of 3-methylcrotonyl CoA carboxylase deficiency. Mol Genet Metab 2008;93:363-70.

2. Baumgartner MR, Almashanu $S$, Suormala $T$, Obie $C$, Cole RN, Packman $S$, et al. The molecular basis of human 3-methylcrotonyl-CoA carboxylase deficiency. J Clin Invest 2001;107:495-504.

3. Grünert SC, Stucki M, Morscher RJ, Suormala T, Bürer C, Burda $P$, et al. 3-Methylcrotonyl-CoA carboxylase deficiency: clinical, biochemical enzymatic and molecular studies in 88 individuals. Orphanet J Rare Dis 2012;7:31.

4. Jung $\mathrm{CW}$, Lee BH, Kim JH, Kim GH, Lee J, Choi JH, et al. Uneventful clinical courses of Korean patients with methylcrotonylglycinuria and their common mutations. J Hum Genet 2012;57:62-4.

5. Cho SY, Park HD, Lee YW, Ki CS, Lee SY, Sohn YB, et al. Mutational spectrum in eight Korean patients with 3-methylcrotonyl-CoA carboxylase deficiency. Clin Genet 2012;81:96-8.

6. Ficicioglu C, Payan I. 3-Methylcrotonyl-CoA carboxylase deficiency: metabolic decompensation in a noncompliant child detected through newborn screening. Pediatrics 2006;118:2555-6. 
7. Dirik E, Yiş U, Paşaoğlu G, Chambaz C, Baumgartner MR. Recurrent attacks of status epilepticus as predominant symptom in 3-methylcrotonyl-CoA carboxylase deficiency. Brain Dev 2008;30:218-20.

8. Baykal T, Gokcay GH, Ince Z, Dantas MF, Fowler B, Baumgartner MR, et al. Consanguineous 3-methylcrotonyl-CoA carboxylase deficiency: early-onset necrotizing encephalopathy with lethal outcome. J Inherit Metab Dis 2005;28:229-33.

9. Oude Luttikhuis HG, Touati G, Rabier D, Williams M, Jakobs C, Saudubray JM. Severe hypoglycaemia in isolated 3-methylcrotonyl-CoA carboxylase deficiency; a rare, severe clinical presentation. J Inherit Metab Dis 2005;28:1136-8.

10. Fonseca $H$, Azevedo L, Serrano $C$, Sousa $C$, Marcão A, Vilarinho L. 3-Methylcrotonyl-CoA carboxylase deficiency: mutational spectrum derived from comprehensive newborn screening. Gene 2016;594:203-10.

11. 1000 Genomes Project Consortium, Auton A, Brooks LD, Durbin RM, Garrison EP, Kang HM, et al. A global reference for human genetic variation. Nature 2015:526:68-74.

12. Stenson PD, Ball EV, Mort M, Phillips AD, Shiel JA, Thomas NS, et al. The Human Gene Mutation Database (HGMD $\left.{ }^{\circledR}\right): 2003$ update. Hum Mutat 2003;21:577-81.

13. Lek M, Karczewski KJ, Minikel EV, Samocha KE, Banks E, Fennell T, et al. Analysis of protein-coding genetic variation in 60,706 humans.
Nature 2016:536:285-91.

14. Dantas MF, Suormala T, Randolph A, Coelho D, Fowler B, Valle D, et al. 3-Methylcrotonyl-CoA carboxylase deficiency: mutation analysis in 28 probands, 9 symptomatic and 19 detected by newborn screening. Hum Mutat 2005;26:164

15. Lam C, Carter JM, Cederbaum SD, Neidich J, Gallant NM, Lorey F, et al. Analysis of cases of 3-methylcrotonyl CoA carboxylase deficiency (3MCCD) in the California newborn screening program reported in the state database. Mol Genet Metab 2013;110:477-83.

16. Cho KL, Kim YJ, Yang SH, Kim GH, Lee JH. Maternal 3-methylcrotonylcoenzyme A carboxylase deficiency with elevated 3-hydroxyisovalerylcarnitine in breast milk. Korean J Pediatr 2016; 59(Suppl 1):S41-4.

17. Rips J, Almashanu S, Mandel H, Josephsberg S, Lerman-Sagie T, Zerem A, et al. Primary and maternal 3-methylcrotonyl-CoA carboxylase deficiency: insights from the Israel newborn screening program. J Inherit Metab Dis 2016;39:211-7.

18. Lehnert W, Niederhoff $H$, Suormala $T$, Baumgartner ER. Isolated biotin-resistant 3-methylcrotonyl-CoA carboxylase deficiency: longterm outcome in a case with neonatal onset. Eur J Pediatr 1996;155: $568-72$.

19. Thomsen JA, Lund AM, Olesen JH, Mohr M, Rasmussen J. Is L-Carnitine supplementation beneficial in 3-Methylcrotonyl-CoA carboxylase deficiency? JIMD Rep 2015;21:79-88. 